\section{PPPL-1707}

$\mathrm{uC}-20 \mathrm{~b}, \mathrm{c}, \mathrm{f}$

\title{
DISRUPTIVE SHIFT OF MAGNETIC AXIS IN NON-CIRCULAR TOKAMAKS
}

BY

$M$, OKABAYASHI, $H$, MAEDA,

$H$, TAKAHASHI, AND M. REUSCH

\section{PLASMA PHYSICS LABORATORY}

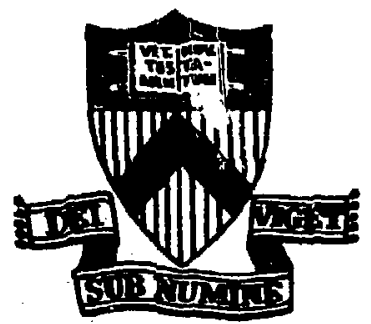

MSTRIDUTLOA OC THS BOCUHENT IS UHLIMTED

\section{PRINCETON UNIVERSITY PRINCETON, NEW JERSEY}

This work was supported by the y.5. Deportment of Energy Contraet HO. DE-ACO2-76-CHO 3073. Reproduetion, Iransiztion, publication, use and disposal, ir. whole or in part. by or for the United States government is permitied. 
Disruptive Shift of Magnetic Axis in Non-Cixcular Tokamaks

M. Okabayashi, H. Maeda, H. Takahashi and M. Reusch

\author{
Princeton University, Plasma Physics Laboratory \\ Princeton, New Jersey 08544
}

\begin{abstract}
Tho magnitude of magnetlc axis shift, following a sudden, disruptive decrease in Internal Inductance and poloidal $B$, can be greately reduced in non-circular tokamaks by exploiting the negative decay index of the equilibrium magnetic field. In this sense a non-circular plasma has important advantages over a circular plasma in preventing the catastrophic consequences of major disruptions. A tokamak design and operational concept is discussed, which exploits these advantages, and is fundamentally different from the tradtional approach.
\end{abstract}

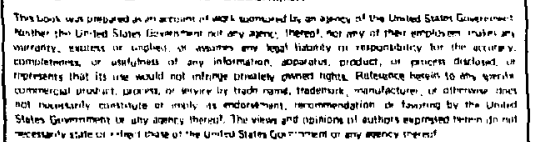

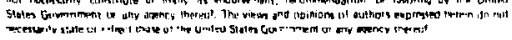




\section{INTRODUCTION}

It has been reported $[1,3]$ that a disruption begins when a sudden rearrangement of the plasma current distribution reduces the internal inductance and poloidal $B$. The resultant rapid inward radial shift of the plasma column often leads to the total annihilation of the plasma current. The rapidly changing plasma current may generate high transient voltages in coil-power supply systems and may induce large eddy currents in various components of the tokamak system, which, in turn, interact with the magnetic fields to produce large forces acting on the components. These effects, which have hitherto been insignificant in small tokamaks built to date, are expected to pose severe constraints on tokamak reactor design.

Intensive experimental and theoretical efforts [1-4] have been made to investigate physical mechanisms that lead to this sudden current rearrangement. These investigations attempt to obtain detailed understanding of the interactions of the plasma with the magnetic fields. In contrast, this paper examines the possibility of minimizing the radial shift, once the current rearrangement takes place, using purely equilibrium MHD considerations. The authors believe that this is probably the first paper to suggest that the magnetic configurations inherent to non-circular tokamak plasmas can be exploited to minimize the inward radial shift and thus help to prevent the catastrophic consequences of major plasma disruptions.

\section{ANALYSIS}

Insight into the advantage of the non-circular configurations can be obtained through the following crude analysis. The plasma current ( $I_{p}$ ), major 
(R) and minor (a) radii and the equilibrium vertical magnetic field (B), are related through Shafranov equilibrium equation,

$$
\begin{aligned}
4 \pi B_{E} R & =\mu_{\Delta} I_{p}\left\lfloor\log \frac{8 R}{a}-\frac{3}{2}+\Delta\right\rfloor \\
\Delta & \equiv \frac{\ell_{i}}{2}+B_{\theta}
\end{aligned}
$$

where $l_{i}$ and $b_{\theta}$ are the dimensionless internal inductance and the ratio of the thermal tc. poloidal magnetic pressure, respectively. Upon taking the total derivative, $D / D t=\partial / \partial t+v \partial / \partial R$, of Eq. (1), we obtain,

$$
R V \frac{\partial B_{E}}{\partial R}+B_{E} \frac{\partial R}{\partial t}=\frac{I_{P}}{4 \pi}\left\lfloor\frac{1}{R} \frac{\partial R}{\partial t}+\frac{D \Delta}{D t}\right\rfloor
$$

where we assumed for simplicity that the plasma current and the equilibrium field are time-invariant. (Removal of these assumptions does not change the conclusions in substantial manner.) When a sudden decrease in the internal inductance and poloidal B takes place, the plasma will shift inward until a new equilibrium position is Eound. Let a change in the various quantities from just before to just after such a shift be represented by the symbol $\delta$. If we appraximate the plasma radial velocity and change in $\Delta$ by $D R / D t=\delta R / \delta t$ and $D \Delta / D t \approx \delta \Delta / \delta t$, respectively, we obtain for the radial shift,

$$
\frac{\delta \delta_{R}}{R}=\frac{\delta \Delta}{(1-n)(\log 8 R / a-3 / 2+\Delta)-1}
$$


where the magnetic field decay index, $n$, is defined by,

$$
\mathrm{n} \equiv-\frac{\mathrm{R}}{\mathrm{B}_{\mathrm{E}}} \frac{\partial \mathrm{B}_{\mathrm{E}}}{\partial \mathrm{R}} \text {. }
$$

Figures 1 and 2 show the magnetic surfaces and equilibrium vertical magnetic fields for typical circular and non-circular (divertor) configurations obtalned for the PDX tokamak. The corresponding radial variations of the magnetic decay index (n-index) are shown in Figs. 3 and 4 For these configurations. For the circular case (Fig. 3) tile n-index is positive and approximately constant over nearly the entire major radius range that falls within the vacuum vessel. For the non-circular configuration (Fig. 4) the $n$-index varies from +0.5 to -2.5 as the major radius varies from 1.4 to $1.1 \mathrm{~m}$. In the region of negative n-index the plasma cross-section is noncircular (Fig. 2). Let us compare the magnetic axis shift expected for these two configurations when the current distribution changes from a parabolic to uniform profile with an accompanying decrease of $\delta \Delta=0.25$ in the internal inductance. Equation (2) yields a $5 \mathrm{~cm}$ shift from $\mathrm{R}=1.15 \mathrm{~m}$ to $1.10 \mathrm{~m}$ for the non-circular plasma with $n=-1.5$, while the circular plasma with $n=$ +0.28 is expected to move as much as $34 \mathrm{~cm}$ Erom $R=1.38 \mathrm{~m}$ to $1.04 \mathrm{~m}$. In the non-circular configuration the magnetic axis will remain in the neighborhood of the original location after a sudden current redistribution. The radial position of the non-circular plasma is therefore much more amenable to control than that of the circular plasma.

We have so far neglected to mention that the non-circular plusma with its 
negative $n$-index is unstable agdinst vertical displacement. Recent experimental results [5] show, however, that this MHD instability can be stabilized over a wide range of the n-index values by means of eddy curronts in coils and their casings. In this passively stabilized regime the plasma moves slowly with a resistive decay time of the eddy currents (several tens of railliseconds), and is quite amenable to external feedback control. When the n-index is too negative, however, the passive stabilization is no longer effective enough and the plasma is rapidly displaced vertically with a MHD time scale (a few microseconds). These two regimes, passively stabilized and MHD instable, are indicated in Fig. 4. The passively stabilized regime extends over a large major radius range for the PDx device. It should be possible to make the range even greater when tokamak design is specifically optimized with this respect. we propose that tokamaks be designed and operated in this regime with a suitable external means of vertical position control in order to obtain a significant improvement in the radial stability. These properties of the non-circular sonfiguration to resist radial displacement are studied further by numerical computations. The first step of the computation is to find a plasma current distribution which gives a typical experimental value of the internal inductance and poloidal $B$ in the range, $\Delta=0.85-1.0$. The starting major and minor radil are $1.38 \mathrm{~m}$ and $0.38 \mathrm{~m}$, respectively. In the second step the magnetic axis is moved inwara by making the current distribution more and more flat until $\Delta$ is reduced by $\delta \Delta=0.25$. The external magnetlc fields are hald constant during this process. The results for the circular cross section indicate that the magnetic axis should vary from $R=1.38 \mathrm{~m}$ to $1.03 \mathrm{~m}$, in good agreement with the earlier estimate. However, in the actual PDX device the plasma is bounded by protective limiters located at $R=0.85 \mathrm{~m}$. Therefore, we expect that the plasma strikes the 
Iimiters before reaching $R=1.03 \mathrm{~m}$ and establishes a new equilibrium location around $\mathrm{R}=1.2 \mathrm{~m}$ with a smaller minor radius.

In the non-circular configuration the n-index at the starting position (point A in Fig. 4 ) is positive. The plasma then moves to $\mathrm{R}=1.27 \mathrm{~m}$ (point B) after a loss of 0.25 in $\Delta$. We assure that the plasma gradualiy recovers a more peaked current profile while remaining stationary at this location under the influence of the ohmic heating and equilibrium fields (point $C$ ). When the current distribution becomes sufficiently peaked, another disruption occurs and the same process is repeated (points C, D and E). The plasma moves to successively smaller major radii with more negative values of the n-index (such as points B, D, and F). The amount of the computed shift in the last step, from $R=1.18$ to $1.13 \mathrm{~m}$, is in reasonable agreement with the earlier analytical estimate. In the absence of a neans of radial position control, the plasma eventually moves into the MHD unstable region and is rapidly lost due to a large vertical displacement.

\section{DISCUSSIONS}

The tokamaks have been traditionally designed with the $n$-index values in the range of $0<n<1$ to assure both radial and vertical stability. Little consideration has been given to possible large plasma motion for this $\mathbf{t}_{\text {. }}$ index range. The foregoing analysis, however, points to a fundamentally different design and operational approach. Tokamaks may be designed with the n-index in moderately negative range, say, $-2<n<0$, to greatly reduce tha radial shift of the plasma position in the event of a disruption. The radial position may then be held within a narcow range by means of an active external control. The vertical instability associated with the negative n-index may be 
damped by passive stabilizing coils and the vertical position further controlled by a feedback system.

Some characteristics of the poloidal field power supply systems desirable for such a tokamak design may now be en.imerated. In order to hold the plasma radial position steady in the face of a sudden decrease of the internal inductance, the equilibrium field must be reduced quickly. This suggests the desirability of a power supply which can swing the current rapidly in either direction. In fact, an ability to reduce the current rapidly is more important than an ability to increase the current, and the supply may be built asymmetrically in this respect, if any engineering or cost advantages can be gained by coing so.

Another important phenomenon during a discuption is an increase of plasma resistivity, which is currently attributed to a substantial increase of impurity influx and/or to ergodic magnetic surface structure. This increasc in plasma resistivity causes a rapi! decrease of the plasma current and a greatly decreased plasma ring L/R-time. In this respect, incorporating in the tokamak design a separate, fast-acting, ohmic heating system which can be triggered in the event of a disruption may greatly help the plasma to replenish the magnetLc flux and thermal energy lost during the disruption.

\section{CONCLUSIONS}

It is shown that the discuptive shift of the magnetic axis, accompanied by a sudden decrease of the internal inductance and poloidal $B$, is nuch smaller for non-circular tokamak configurations than for circular configurations. The proposed tokamak design and operational concept, Eundamentally different from the traditional one, exploits this fact to help 
prevent the catastrophic consequences of disruptions. Such tokamaks have the following characteristics.

(1) The decay index of the equilibrium vertical magnetic field is in a moderately negasive regime, for example, $-2<n<0$, over a wide range of the major radius,

(2) fast-acting equilibrium field power supply, possibly with greater capability to reduce the current rapidly than to increase the current, iE engineering and cost advantages can be gained, and

(3) a separate, fast-acting ohmic heating supply system that can be triggered in the event of a disruption, to help the plasma to replenish the magnetic $f l u x$ and thermal energy lost.

\section{ACKNOWIJEDGMENT}

The authors are indebted to $\mathrm{K}$. Bol and D. Meade for various discussions. This work was supported by the United States Department of Energy Contract No. DE-AC02-76-CHO-3073. 


\section{REFERENCES}

[1] G. aATEMAN, MHD Instabilities, MIT Press (1978) 226.

[2] A. SYXES and J. WESSEn, Phys. Rev, Lett, 44 (1980) 1215.

[3] R. WhITE, D. MONTICELlo, and M. Rosenbluth, Phys. Rev. Lett. 39 (1977) 1618.

(4) K. MCGUIRE and D. ROBINSON, Phys. Rev. Lett $44(1990) 1666$.

[5] D. MensE, et al., in Proc. Plasma Phys. and Cont. Nucl. Fusion Res., Brussels, 1980. 

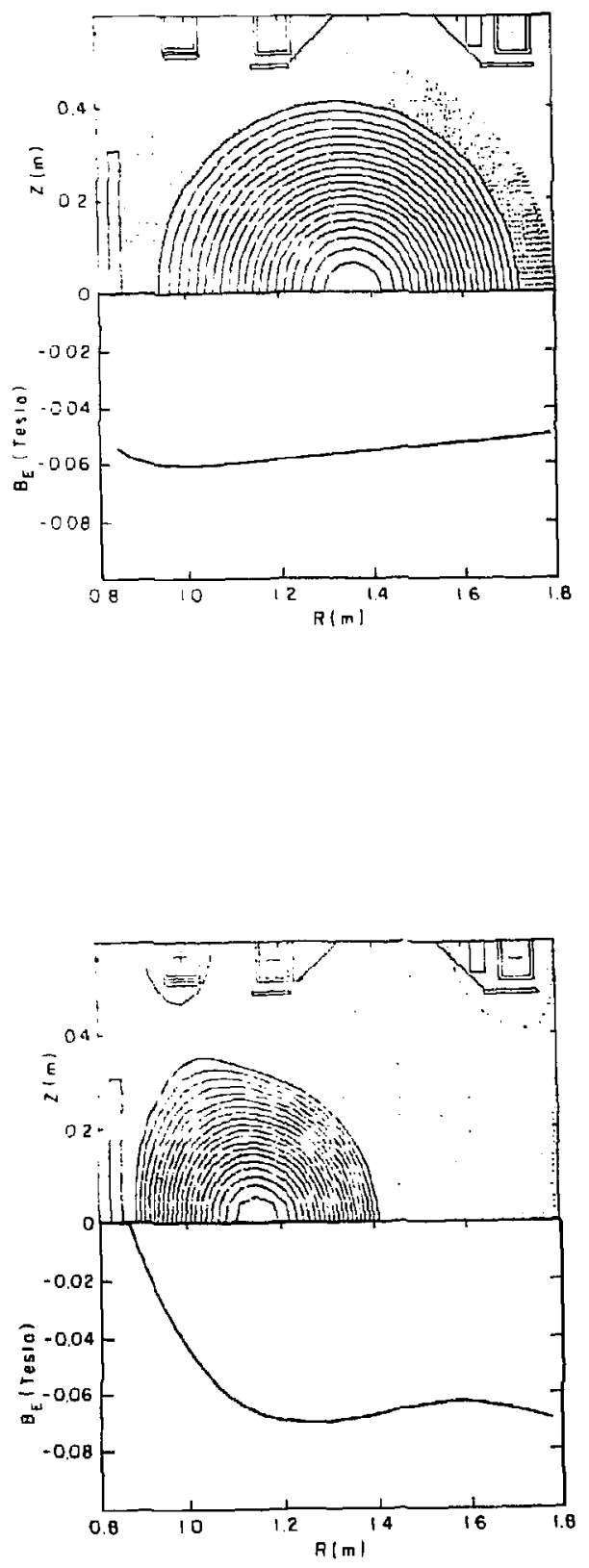

(PPPL-806259)

Fig. 1. Magnetic surfaces and equilibrium vertical magnetic field of PDX tokanak in circular plasma configuration. (With $\mathrm{I}_{\mathrm{p}}=$
$300 \mathrm{kA}$ )
(PPE L-806260)

Fig. 2. Magnetic surfaces and cquilibrilm vertical magnetic field of PDX tokamak in non-circular plasma configuratior. (With $T_{D}$, $300 \mathrm{kA}$ ) 


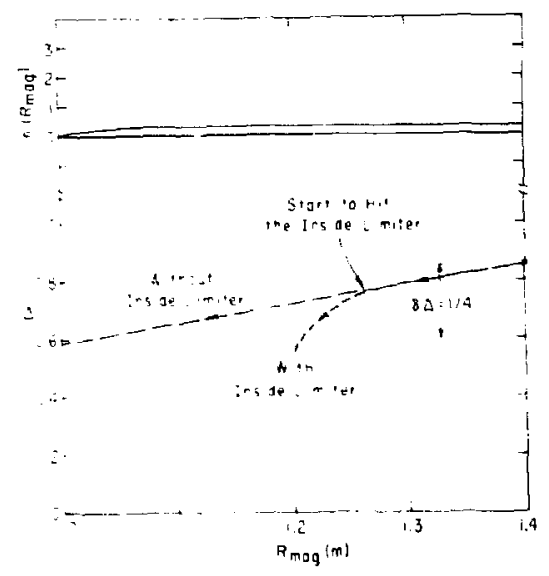

(PPPL-806115)

Fig. 3. The decay index of equilibrium field and the trajectory in internal inductance -- major radius parameter space during disruption in circular plasma conEiguration.

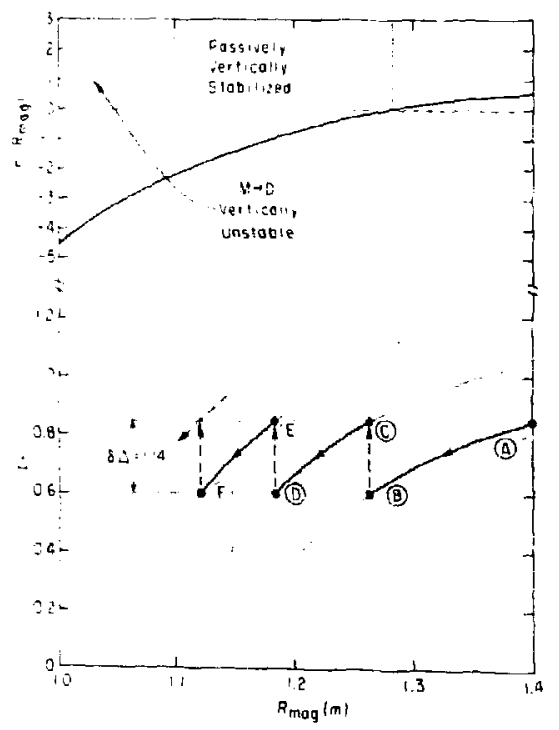

(PPPL-806116)

Fig. 4. The decay index of equilibrium field and the trajectory in internal inductance -- major radius parameter space during a series of disruptions in noncircular plasma configuration. 